SERIE B - INFORMATIK

\title{
A Subexponential Algorithm for Abstract Optimization Problems
}

\author{
Bernd Gärtner
}

B $93-05$

May 1993

\begin{abstract}
An Abstract Optimization Problem (AOP) is a triple $(H,<, \Phi)$ where $H$ is a finite set, $<$ a total order on $2^{H}$ and $\Phi$ an oracle that, for given $F \subseteq G \subseteq H$, either reports that $F=\min _{<}\left\{F^{\prime} \subseteq G\right\}$ or returns a set $F^{\prime} \subseteq G$ with $F^{\prime}<\bar{F}$. To solve the problem means to find the minimum set in $H$. We present a randomized algorithm that solves any AOP with an expected number of at most $e^{2 \sqrt{n}+O(\sqrt[4]{n} \ln n)}$ oracle calls, $n=|H|$. In contrast, any deterministic algorithm needs to make $2^{n}-1$ oracle calls in the worst case.

The algorithm is applied to the problem of finding the minimum distance between two $n$-vertex (or $n$-facet) polyhedra in $d$-space, and to the computation of the smallest ball containing $n$ points in $d$-space; for both problems we give the first subexponential bounds in $d$.

KEYWORDS: computational geometry, smallest enclosing ball, minimum distance between polyhedra, local optimization, randomized algorithm.
\end{abstract}

\footnotetext{
*Graduiertenkolleg "Algorithmische Diskrete Mathematik", Freie Universität Berlin, Fachbereich Mathematik, Institut für Informatik, Takustr. 9, W-1000 Berlin 33, e-mail: gaertner@inf.fu-berlin.de † Supported by the Deutsche Forschungsgemeinschaft (DFG) under grant We 1265/2-1, and by the ESPRIT Basic Research Action Program 7141 of the EC (ALCOM II). A preliminary version appeared in Proc. 33rd Annual IEEE Symposium on Foundations of Computer Science (1992), 464-472.
} 


\section{Introduction}

Three geometric optimization problems. Recently, Sharir \& Welzl [SW] have described an abstract class of problems - so-called LP-type problems - that are efficiently solvable by a simple randomized algorithm. The typical LP-type problems are geometric optimization problems, in spirit related to the 'master' problem of Linear Programming:

(LP) Given a polyhedron $\mathcal{P}$, specified by $n$ halfspaces in $d$-space, and a $d$-vector $v$, find a point $p \in \mathcal{P}$ extreme in direction $v$.

The geometric formulation is chosen in order to keep notation consistent with another important LP-type problem, namely finding the minimum distance between polyhedra:

(POLYDIST) Given two polyhedra $\mathcal{P}$ and $\mathcal{Q}$, specified by $n$ points (or $n$ halfspaces) in $d$-space, find points $p \in \mathcal{P}, q \in \mathcal{Q}$ with $\|p-q\|=\operatorname{dist}(\mathcal{P}, \mathcal{Q})$.

The minimum spanning ball problem looks somewhat different, but nevertheless fits into the LP-type framework:

(MINIBALL) Given $n$ points in $d$-space, determine the center and radius of the smallest ball containing all the points.

LP-type problems can be solved in a local optimization fashion, i.e. if a proposed solution is not optimal yet, one can locally improve on it (by solving a 'small' subproblem). This basic property underlies the algorithm of [SW]; as shown in [MSW], its combinatorial time complexity (i.e. in the unit-cost model) is subexponential in $d$ for LP. This is due to the fact that - at least under certain standard assumptions - the small instances $(n=d+1)$ can easily be solved in polynomial time. For POLYDIST and MINIBALL, however, the small problems become an issue, and all known combinatorial bounds are exponential in $d$, so that the analysis of [MSW] does not carry over to these problems.

In this paper we will show that the subexponential bound for LP is actually induced only by the local optimization property and does not rely on additional convenient features of the small instances. Consequently, POLYDIST and MINIBALL can be solved as efficiently as LP by our method. The tool is the framework of the Abstract Optimization Problems (AOPs) that captures the spirit of local optimization in a generic setting.

Basically, an AOP consists of a finite set $H$ with a total order on $2^{H}$ and an oracle that answers the following queries: for given $F \subseteq G \subseteq H$, does there exist a set $F^{\prime} \subseteq G$ with $F^{\prime}<F$ ? If the answer is yes, such a set is returned as a witness. To solve the problem means to find the minimum set in the total order, and we want to bound the number of oracle queries needed to do this for any given AOP.

The algorithm we develop is randomized, i.e. the expected number of oracle queries is averaged over internal coin flips performed by the algorithm and is independent of the input. Moreover, randomization is crucial: if information about the linear order $<$ can be obtained from the oracle only, no deterministic algorithm can beat the trivial bound of $2^{|H|}-1$ oracle queries in the worst case. Although this does not mean much for a specific instance of an AOP, it gives evidence that randomized algorithms may be potentially more powerful than deterministic ones in this situation.

The paper is organized as follows: in the rest of the introduction we give a brief survey on results concerning the combinatorial complexity of the LP, POLYDIST and MINIBALL problem; in Section 2 we will discuss Sharir \& Welzl's LP-type problems and point out why the subexponential bound for LP established in [MSW] does not hold for POLYDIST and MINIBALL. In Section 3 we formally introduce our abstract framework and state the main result of the paper that implies the subexponential bounds for POLYDIST and MINIBALL. Section 4 contributes the more technical part; it proves the deterministic lower bound and the randomized upper bound by presenting an algorithm for the abstract framework. Section 5 provides a concluding discussion. 
Linear Programming. LP problems are probably the best-understood optimization problems. There exists a vast amount of literature; the reader is referred to [Sch] for an introduction. There are several methods to solve LP-problems that are efficient in practice - the most popular one being the simplex algorithm that was introduced by Dantzig [Dan] in 1951.

The simplex algorithm, however, is of a heuristic nature, i.e. it behaves much better in practice than what one can prove. For a long time it was even open whether LP-problems can be solved in polynomial time (in the number $d$ of variables and the number $n$ of constraints). This was settled by Khachiyan [Kha] and Karmarkar [Kar], but their algorithms are not combinatorial, i.e. they do not only depend on $d$ and $n$ but also on the size of the coefficients defining the input. It is still open whether there exists a combinatorial algorithm with polynomial complexity. In particular, the simplex algorithm was shown to be exponential in $d$ in the worst case for many pivot rules [KM].

Nevertheless, there has been substantial progress on the worst case complexity of combinatorial algorithms for Linear Programming over the last years. Megiddo [Meg] was the first to show that LP can be solved in time linear in the number of constraints. For improvements in the dependence on $d$ see [Dye1], [Cla1], [Sei], [CM].

The currently best randomized algorithm combines the recent subexponential results independently obtained by Kalai [Kal] and Matoušek, Sharir \& Welzl [MSW] with an algorithm by Clarkson [Cla2]. This gives a subexponential bound of

$$
O\left(d^{2} n+e^{O(\sqrt{d \log d})}\right) .
$$

Note that the best deterministic algorithm is obtained by 'derandomizing' Clarkson's algorithm and has runtime $O\left(d^{O(d)} n\right)$, which is still exponential in $d[\mathrm{CM}]$.

Minimum Distance between Polyhedra. This problem - and the important special case that one polyhedron is a single point - has applications e.g. in motion planning (collision testing), and there are heuristics for it without time analysis (see e.g. Wolfe [Wol] for the special case, Sekitani \& Yamamoto [SY] for the general case). A first nontrivial randomized time bound of $O\left(n^{\lfloor/ 2\rfloor}\right.$ ) was given by Clarkson [Cla3] (provided the polyhedra are specified by $n$ points). Applying the algorithm in [SW], this can be improved to give the so far best bound of $O\left(d^{3} 2^{d} n\right)$, which is linear in $n$ but still exponential in $d$. Our algorithm will establish the same subexponential bound as stated above for Linear Programming.

Minimum Spanning Ball. It was observed early that MINIBALL (which is a prototype problem in facility location) has a structure similar to LP and thus can also be solved in time linear in the number of points by the techniques in [Meg] and [Dye1] (with the same dependence on $d$ as in the case of $\mathbf{L P}$ ). As observed by Welzl [Wel], the $\mathbf{L P}$ algorithm of Seidel [Sei] also applies to MINIBALL, and the same holds for the algorithms of Clarkson and Sharir \& Welzl. As in the case of POLYDIST, subexponential runtime could not yet be shown but will be established in this paper.

\section{Basics and Terminology}

LP-type problems. To begin with, let us briefly review the concept of the LP-type problems introduced in [SW], where the reader can also find how $\mathbf{L P}$ itself fits into the framework. Consider the MINIBALL problem first, and let $H$ be a set of $n$ points in $d$-space. For $G \subseteq H$ denote by $w(G)$ the radius of the smallest ball containing the points in $G$. It is well known that this ball is unique and that for $e \in H, w(G)<w(G \cup\{e\})$ if and only if $e$ lies outside the ball determined by $G$. From this it is easily seen that the following two properties hold for all $F \subseteq G \subseteq H$ :

(i) $w(F) \leq w(G)$.

(ii) If $w(F)=w(G)$, then $w(F)<w(F \cup\{e\}) \Leftrightarrow w(G)<w(G \cup\{e\})$, for all $e \in H$.

In general, any pair $(H, w)$ satisfying (i) and (ii) is called an LP-type problem. A basis is a set $B \subseteq H$ with $w\left(B^{\prime}\right)<w(B)$ for all $B^{\prime} \subsetneq B$. A basis of $G \subseteq H$ is a basis $B \subseteq G$ such that 


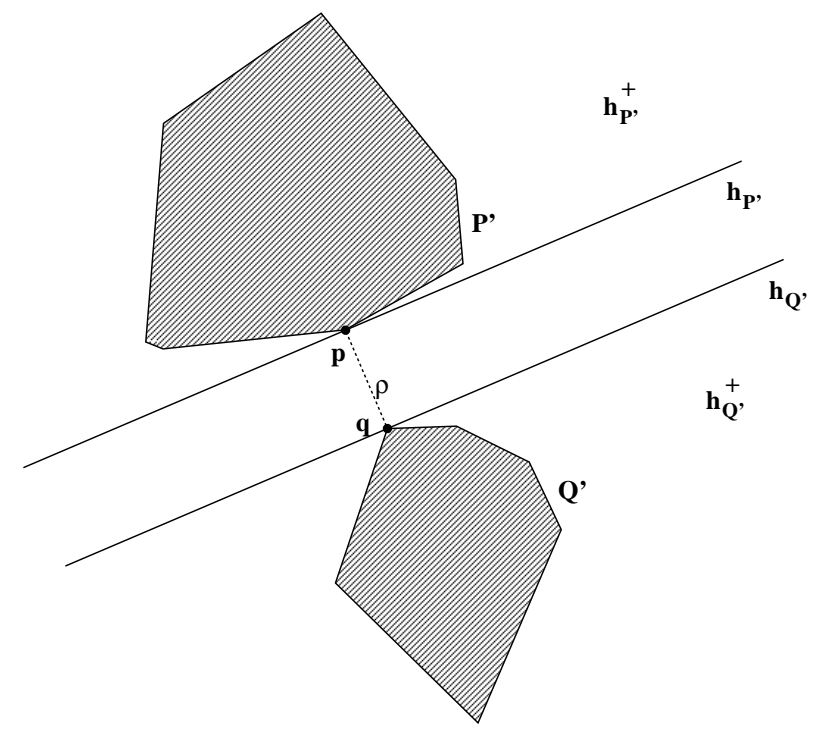

Figure 1: The POLYDIST problem

$w(B)=w(G)$. The combinatorial dimension of $(H, w)$ is the maximum cardinality of any basis. In this framework, MINIBALL has combinatorial dimension at most $d+1$, because any minimum ball spanned by a set $G \subseteq H$ is already determined by at most $d+1$ points of $G$ on the boundary.

POLYDIST gives rise to an LP-type problem as follows: we assume for the rest of the paper, that polyhedra $\mathcal{P}$ and $\mathcal{Q}$ are given by point sets $P$ and $Q, P \cap Q=\emptyset,|P \cup Q|=n$. So $\mathcal{P}$ and $\mathcal{Q}$ are actually polytopes with $\mathcal{P}=\operatorname{conv}(P), \mathcal{Q}=\operatorname{conv}(Q)$ (the case where $\mathcal{P}$ and $\mathcal{Q}$ are specified by halfspaces is similar but requires more technicalities).

For $P^{\prime} \cup Q^{\prime} \subseteq P \cup Q$, let

$$
w\left(P^{\prime} \cup Q^{\prime}\right):=\operatorname{dist}\left(\operatorname{conv}\left(P^{\prime}\right), \operatorname{conv}\left(Q^{\prime}\right)\right) .
$$

For $P^{\prime}$ or $Q^{\prime}$ empty, $w$ is set to $\infty$. Now properties (i) and (ii) (for $w(F)=w(G)<\infty$ ) hold for the pair $(P \cup Q, w)$, but with ' $<$ ' and ' $\leq$ ' replaced by ' $>$ ' and ' $\geq$ ', respectively. Property (i) is obviously satisfied, and property (ii) easily follows from the following fact: assume $w\left(P^{\prime} \cup Q^{\prime}\right)=\rho, 0<\rho<\infty$. Then there exists a unique pair $h_{P^{\prime}}, h_{Q^{\prime}}$ of parallel supporting hyperplanes of distance $\rho$ which are perpendicular to any vector $p-q$ with $p \in \operatorname{conv}\left(P^{\prime}\right), q \in \operatorname{conv}\left(Q^{\prime}\right)$ and $\|p-q\|=\rho$.

Denote by $h_{P^{\prime}}^{+}$and $h_{Q^{\prime}}^{+}$, the closed halfspaces containing $P^{\prime}$ and $Q^{\prime}$, respectively. For a point $e \in P-P^{\prime}\left(Q-Q^{\prime}\right)$ we have $w\left(P^{\prime} \cup Q^{\prime} \cup\{e\}\right)<w\left(P^{\prime} \cup Q^{\prime}\right)$ if and only if $e$ does not lie in $h_{P^{\prime}}^{+}$ $\left(h_{Q^{\prime}}^{+}\right)$(Figure 1$)$.

It is a straightforward exercise to show that the following holds:

Lemma 1 In the LP-type framework, the combinatorial dimension of POLYDIST defined by polytopes $\mathcal{P}$ and $\mathcal{Q}$ in $d$-space satisfies $\delta \leq d+2$ (if $\mathcal{P}$ and $\mathcal{Q}$ are disjoint, $\delta \leq d+1$ ).

Here is the main result of $[\mathrm{MSW}]^{1}$ :

Theorem 2 Let $(H, w)$ with $|H|=n$ be an LP-type problem of combinatorial dimension $\delta$, and denote by $t_{\text {small }}$ the time necessary to compute a basis of $G$ and its value $w(G)$ for $|G|=\delta+1$. Then a basis of $H$ and $w(H)$ can be computed in time

$$
O\left(t_{\text {small }} n e^{2 \sqrt{\delta \ln n}}\right) .
$$

\footnotetext{
${ }^{1}$ The bounds are actually slightly better (and more complicated) than what we cite here.
} 
As the theorem shows, the time bound for an LP-type problem crucially depends on the complexity of the 'small' instances, and it is not clear that solving them is a substantially easier task than solving the whole problem. For $d$-dimensional LP, the small problem basically consists of solving a linear program with $d+1$ constraints, which can easily be done in time polynomial in $d$ by observing that the solution is always determined by exactly $d$ of the constraints (provided, the problem is bounded), so that it suffices to test all $d$-subsets of the constraint set for optimality (for details see [MSW] ).

In case of POLYDIST and MINIBALL, the small problems are

(SMALL_POLYDIST) Given two polyhedra $\mathcal{P}$ and $\mathcal{Q}$, specified by at most $d+3$ points $P \cup Q$ in $d$-space, determine their minimum distance and a basis, i.e. a minimal subset $P^{\prime} \cup Q^{\prime}$ determining the same distance.

and

(SMALL_MINIBALL) Given a set $H$ of at most $d+2$ points in $d$-space, determine the radius of the smallest ball containing $H$ and a basis, i.e. a minimal subset $B$ determining the same ball.

In contrast to $\mathbf{L P}$, it is no longer true that a basis has fixed cardinality, even in the generic situation; in case of POLYDIST, a basis of $P \cup Q$ may consist of any number of points between 2 and $d+2$. Consequently, straightforward checking of every candidate basis as in the case of LP may require the examination of $\Theta\left(2^{d}\right)$ subsets just to solve SMALL_POLYDIST, which gives nothing better than an exponential algorithm for the whole problem. The same difficulty arises in the MINIBALL problem: a minimum spanning ball may be determined by any number of points between 2 and $d+1$ on its boundary, so again SMALL_MINIBALL is not efficiently solvable by the trivial method. By embedding SMALL_POLYDIST into the AOP framework we will be able to solve it in time $e^{O(\sqrt{d})}$, and the same complexity will be achieved for SMALL_MINIBALL, which will turn out to be a special case of SMALL_POLYDIST. Plugging this into Theorem 2 will give subexponential bounds also for the corresponding 'large' problems.

\section{Abstract Optimization Problems}

Let us repeat the definition of an AOP in a formal way in order to have the accurate terminology available.

Definition 3 An $A O P$ is a triple $(H,<, \Phi)$ where $H$ is a finite set, $<$ is a total order on $2^{H}$, and $\Phi$ is a mapping

$$
\Phi: \mathcal{H} \rightarrow H, \mathcal{H}:=\{(F, G) \mid F \subseteq G \subseteq H\}
$$

with the following property:

$$
\begin{aligned}
\Phi(F, G) & =F \text { if and only if } F=\min _{<}\left\{F^{\prime} \subseteq G\right\}, \\
G \supseteq \Phi(F, G) & <F \text { otherwise. }
\end{aligned}
$$

For $G \subseteq H$ let $\operatorname{opt}(G)$ denote $\min _{<}\{F \subseteq G\}$. Solving the AOP means to find opt $(H)$.

We achieve the following results (proofs are postponed to the following technical section):

Theorem 4 (Deterministic lower bound) For any deterministic algorithm $\mathcal{A}$ that solves all AOPs on a set $H,|H|=n$, there exists an $A O P(H,<, \Phi)$ that cannot be solved by $\mathcal{A}$ with less than $2^{n}-1$ oracle queries.

Theorem 5 (Randomized upper bound) There exists a randomized algorithm that solves any $A O P$ on a set $H,|H|=n$, with an expected number of at most $e^{2 \sqrt{n}+O(\sqrt[4]{n} \ln n)}$ oracle queries. 


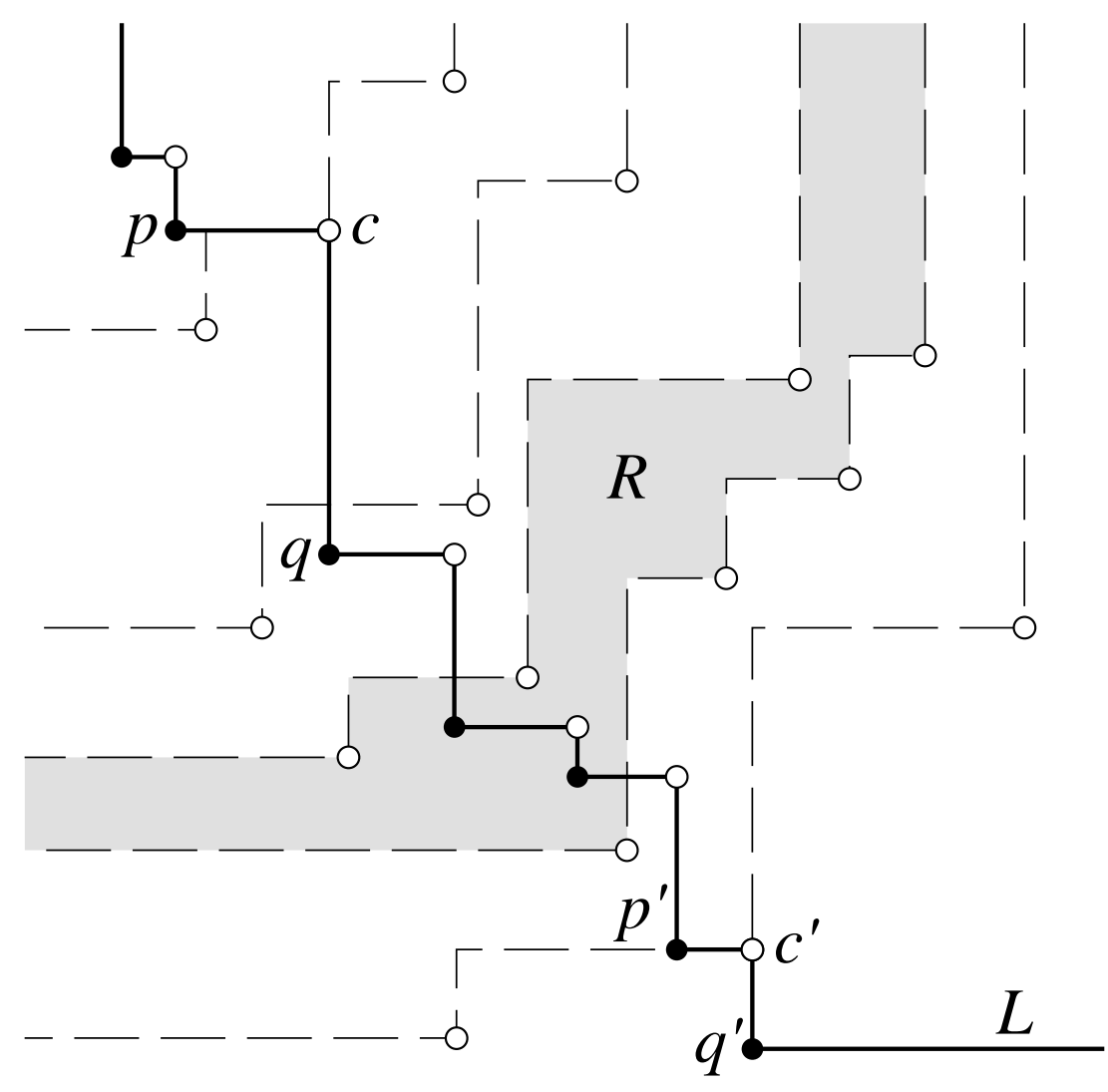

Figure 2: The POLYDIST oracle

Using the terminology from the previous section, we will now demonstrate how POLYDIST defined by point sets $P$ and $Q$ fits into the AOP framework: the ground set $H$ is $P \cup Q$, and the total order $<$ on $2^{H}$ is defined as follows: the bases (in the LP-type sense) are ordered by their $w$-values with ties broken arbitrarily, and any non-basis is larger than any basis. This definition ensures that $\operatorname{opt}(H)$ indeed is a basis of $P \cup Q$ and the non-bases are not of interest.

It remains to describe the oracle $\Phi$. $\Phi$ will only be called on pairs $(F, G)$ where $F$ is a basis and will deliver only bases. We need some more terminology: for a point $p$ and $P^{\prime} \subseteq P$ with $p \in \operatorname{conv}\left(P^{\prime}\right)$ (equivalently for $q, Q^{\prime}$ ) let $f\left(p, P^{\prime}\right) \subseteq P^{\prime}$ be inclusion-minimal with $p \in \operatorname{conv}\left(f\left(p, P^{\prime}\right)\right.$ ). For $F^{\prime}=P^{\prime} \cup Q^{\prime} \subseteq P \cup Q$ let $\left(p_{F^{\prime}}, q_{F^{\prime}}\right)$ be a pair of points realizing the minimum distance between the affine hulls of $P^{\prime}$ and $Q^{\prime}$, i.e. $\left\|p_{F^{\prime}}-q_{F^{\prime}}\right\|=\operatorname{dist}\left(\operatorname{aff}\left(P^{\prime}\right)\right.$, aff $\left(Q^{\prime}\right)$ ). (note that in general neither this point pair nor $f$ is unique). The open region between the two parallel hyperplanes $h_{P^{\prime}} \ni p_{F^{\prime}}$ and $h_{Q^{\prime}} \ni q_{F^{\prime}}$ perpendicular to the vector $p_{F^{\prime}}-q_{F^{\prime}}$ is called the slab of $F^{\prime}$, denoted by $\operatorname{slab}\left(F^{\prime}\right)$ (see Figure 2 (a)).

Now we can implement the oracle $\Phi(F, G)$; its idea is to start with points $p$ and $q$ realizing $w(F)$; provided that one can improve on $F$ at all (which is the case if and only if the slab of $F$ contains a point of $G$ ), a loop is performed in which $p$ and $q$ move along straight lines - thereby decreasing $\|p-q\|-$ until a stable position, i.e. a new basis, is obtained. In the generic step of the loop there are points $p, q$ and sets $P^{\prime}, Q^{\prime}$ such that $p \in \operatorname{conv}\left(P^{\prime}\right), q \in \operatorname{conv}\left(Q^{\prime}\right)$. By definition, $\|p-q\| \geq\left\|p_{F^{\prime}}-q_{F^{\prime}}\right\|$, where $F^{\prime}=P^{\prime} \cup Q^{\prime}$, so by moving $p$ and $q$ simultaneously along straight lines towards $p_{F^{\prime}}$ and $q_{F^{\prime}}$, respectively, their distance decreases in a monotone fashion. The movement stops if either the destination points are reached (in which case $F^{\prime}$ is a new basis) or one of $p$ and 
$q$ hits the boundary of $\operatorname{conv}\left(P^{\prime}\right)$ or $\operatorname{conv}\left(Q^{\prime}\right)$, respectively; in this case, the loop continues after setting $P^{\prime}$ to $f\left(p, P^{\prime}\right)$ and $Q^{\prime}$ to $f\left(q, Q^{\prime}\right)$ which decreases $\left|P^{\prime} \cup Q^{\prime}\right|$ by at least one (Figure $2(\mathrm{~b})$ ). Here is the pseudocode ${ }^{2}$ for the procedure just described:

\begin{tabular}{|ccc|}
\hline$\Phi(F, G)$ & $\triangleright F$ basis, $F=P^{\prime} \cup Q^{\prime} \subseteq P \cup Q=G$ \\
1 & $(p, q):=\left(p_{F}, q_{F}\right)$ & $\triangleright F$ basis $\Rightarrow p_{F}, q_{F} \in \operatorname{conv}\left(P^{\prime}\right), \operatorname{conv}\left(Q^{\prime}\right)$ \\
2 & for all $e \in G-F$ & \\
3 & do if $e \in \operatorname{slab}(F)$ & \\
4 & then case $e$ of \\
5 & $\in P: P^{\prime}-P^{\prime} \cup\{e\}$ \\
6 & $\in Q: Q^{\prime} \leftarrow Q^{\prime} \cup\{e\}$ \\
7 & loop $F^{\prime}-P^{\prime} \cup Q^{\prime}$ \\
8 & $\left(p_{\lambda}, q_{\lambda}\right)-(p, q)+\lambda\left(\left(p_{F^{\prime}}, q_{F^{\prime}}\right)-(p, q)\right)$ \\
9 & $\mu-\max \left\{\lambda \mid p_{\lambda} \in \operatorname{conv}\left(P^{\prime}\right), q_{\lambda} \in \operatorname{conv}\left(Q^{\prime}\right)\right\}$ \\
10 & if $\mu>1$ \\
11 & $\operatorname{then} \operatorname{return} F^{\prime}$ \\
12 & $(p, q)-\left(p_{\mu}, q_{\mu}\right)$ \\
13 & $\left(P^{\prime}, Q^{\prime}\right)-\left(f\left(p, P^{\prime}\right), f\left(q, Q^{\prime}\right)\right)$ \\
14 & return $F$ & \\
\hline
\end{tabular}

From the discussion above, termination of the procedure follows. As far as the correctness is concerned, there is one subtlety: it is not hard to see that only if in some stage of the loop the pair $\left(p_{F^{\prime}}, q_{F^{\prime}}\right)$ happens to be non-unique, line 11 may not return a basis. In this case however, $p_{F^{\prime}}$ can be chosen arbitrarily from an infinite line at least and can therefore be determined to lie outside conv $\left(P^{\prime}\right)$, which implies that the procedure does not enter line 11 in this case. It should be mentioned that $\|p-q\|$ strictly decreases at least during the first iteration of the loop, so the basis finally returned has smaller $w$-value than $F$.

We are interested in the runtime of this oracle, when called on a 'small' problem, i.e. $|G| \leq d+3$. The following bound is rather brute-force; the interesting fact is that it is polynomial.

Lemma 6 Let $G$ be a set of at most $d+3$ points in d-space. The oracle $\Phi(F, G)$ can be implemented to run in time $O\left(d^{5}\right)$.

Proof: The first phase (checking whether an improvement on $F$ is possible) amounts to the computation of one scalar product per point $e \in G-F$ and therefore can be done in time $O\left(d^{2}\right)$. The loop is executed no more than $d+3$ times; in each iteration, points $p_{F^{\prime}}, q_{F^{\prime}}$ can be computed in time $O\left(d^{3}\right)$ by solving a system of linear equalities. In order to find $\mu$, we have to intersect two lines with the bounding hyperplanes of $\operatorname{conv}\left(P^{\prime}\right)$ and $\operatorname{conv}\left(Q^{\prime}\right)$, respectively. From the fact that $F$ was a basis it follows that these polytopes are simplices, so there are no more than $d+1$ bounding hyperplanes per polytope, and each intersection can be computed in time $O\left(d^{3}\right)$ again. This process also gives $f\left(p, P^{\prime}\right)$ and $f\left(q, Q^{\prime}\right)$ as a by-product.

Observe that by arbitrarily choosing two vertices in the beginning, one from each polytope, we get an initial basis for the oracle. Now Theorem 5 states that a SMALL_POLYDIST problem can be solved in time $e^{O(\sqrt{d})}$, and together with Theorem 2 this gives

Theorem 7 The minimum distance between two $n$-vertex polytopes in $d$-space can be computed in time $O\left(n e^{(2+o(1))(\sqrt{d \ln n})}\right)$.

\footnotetext{
${ }^{2}$ The variant we use here is adapted from the book Introduction to Algorithms by T. H. Cormen, C. E. Leiserson \& R. L. Rivest, The MIT Press, 1990
} 
We remark that the same result holds for two $n$-facet polyhedra. Note that the contribution from the small problem is hidden in the o(1) term of the exponent.

We get the same bound for MINIBALL:

Theorem 8 The smallest enclosing ball of a set of $n$ points in d-space can be computed in time $O\left(n e^{(2+o(1))(\sqrt{d \ln n})}\right)$.

For this it suffices to show that a SMALL_MINIBALL problem can be solved as efficiently as SMALL_POLYDIST, and as it turns out, both problems are strongly related; the following correspondence can be found e.g. in [Raj]:

Theorem 9 Let $P$ be a set of $d+1$ affinely independent points in $d$-space with circumcenter $q_{0}$. The center $q_{1}$ of the smallest ball containing $P$ is the point in conv $(P)$ with minimal distance to $q_{0}$.

Thus, in order to solve SMALL_MINIBALL on $d+2$ points, compute - by solving $d+2$ SMALL_POLYDIST problems with one polytope being a single point - all the smallest balls spanned by $d+1$ of the points, and compare their radii. In case the input consists of $d$ points or less, the problem reduces to the affine hull of the points. Note that the circumcenter of $d+1$ points can be computed in time $O\left(d^{3}\right)$.

\section{Bounds for Abstract Optimization Problems}

This section contains proofs of Theorem 4 and Theorem 5. The deterministic lower bound follows from an adversary argument. For the randomized upper bound we present an algorithm along with a careful analysis. Let us start with the lower bound.

\subsection{The Deterministic Lower Bound}

Let $H$ be an $n$-element set and suppose we have a deterministic algorithm to solve any AOP $(H,<, \Phi)$.

We start the algorithm on a problem $\left(H,<_{0}, \Phi_{0}\right)$ with $<_{0}$ and $\Phi_{0}$ not yet determined, and we argue that an adversary answering the oracle queries can construct $<_{0}$ and $\Phi_{0}$ 'online' in such a way that the algorithm is forced to step through at least $2^{n}-1$ queries. When supplied with a query pair $(F, G)$, the adversary will output an answer $F^{\prime}=\Phi_{0}(F, G)$ according to two simple rules:

(i) the answer $F^{\prime}$ is consistent with the previous ones, i.e. there exists an AOP such that the current and all previous queries have been answered correctly with respect to this AOP.

(ii) $F^{\prime}=F$ if and only if there is no other consistent answer.

It is easy to see that the adversary always has a consistent answer, so the algorithm steps through a sequence of queries with pairs $(F, G)$ and finally stops. Suppose that less than $2^{n}-1$ queries have been performed. Then there are two sets $F_{1}$ and $F_{2}$ which have never been the first component of a query pair. We will show that it is consistent with all answers to assume that $F_{1}=\operatorname{opt}(H)$. The same holds for $F_{2}$, so whatever the algorithm outputs, there is an AOP that is not correctly solved. Hence the adversary can force the algorithm to step through at least $2^{n}-1$ queries, which means that it performs that many queries on the AOP that has implicitly been constructed by the adversary.

We are left to prove that $F_{1}=\operatorname{opt}(H)$ is consistent. Clearly, this choice can fail only if some answer has revealed the existence of a smaller set. Since there was no query pair $\left(F_{1}, G\right)$, the only remaining possibility for this to happen is that some query $(F, G)$ with $F_{1} \subseteq G$ has been answered by $F$, thus establishing $F<F_{1}$. But in the first query of this type, $F_{1}$ was not bounded from below yet, so it could have been returned instead of $F$, a contradiction to rule (ii). 


\subsection{The Randomized Upper Bound}

We present a randomized algorithm that solves any given $\operatorname{AOP}(H,<, \Phi)$ on an $n$-element set $H$ with an expected number of at most $e^{2 \sqrt{n}+O(\sqrt[4]{n} \ln n)}$ calls to the oracle $\Phi$. Up to an $O(n)$ overhead caused by set operations, the actual runtime of the algorithm will asymptotically be dominated by the time spent on the oracle calls, so this is a reasonable measure of complexity. The algorithm will eventually output opt $(H)$, but the generic step in the recursive procedure is the computation of opt $(G)$ for $G \subseteq H$. Together with $G$, a set $F \subseteq G$ is maintained; $F$ can be viewed as an 'estimate' for the solution that will be improved over and over again until it coincides with the desired minimum. Our algorithm combines ideas of both Kalai's and Matoušek, Sharir \& Welzl's subexponential LP algorithms and substantially generalizes their applicability.

The section proceeds in stages: in a first stage we introduce a trivial algorithm and the basic terminology; the second stage presents an algorithm that - although it works only modulo a hypothetic subroutine - features the heart of the final algorithm and its subexponential analysis. Stage three describes a 'working' algorithm that will be obtained by 'approximating' the subroutine to a reasonable extent.

Getting Started. Just to get acquainted, let us write down the obvious deterministic method to obtain opt $(G)$ in presence of an estimate $F$ :

\begin{tabular}{|lc|}
\hline Aop_DET $(F, G)$ \\
1 & repeat $F^{\prime}-F$ \\
2 & $F-\Phi(F, G)$ \\
3 & $\operatorname{until} F=F^{\prime}$ \\
4 & $\operatorname{return} F^{\prime}$ \\
\hline
\end{tabular}

In order to solve the problem on $G$, AOP_DET has to call the oracle $2^{|G|}$ times in the worst case, and as we have seen in the previous section, it shares this exponential behavior with any algorithm that is deterministic or calls the oracle only on pairs of the form $(*, G)$. Consequently, the method we will describe now is randomized and uses oracle queries of the form $\Phi\left(*, G^{\prime}\right)$ for certain subsets $G^{\prime} \subseteq G$. It will have one very intuitive property in common with AOP_DET: the better the estimate $F$, the faster the algorithm for $G$. A natural measure for the quality of $F$ in this context is the rank of $F$ with respect to $G$, defined by

$$
\operatorname{rank}(F, G):=\#\left\{F^{\prime} \subseteq G \mid F^{\prime}<F\right\} .
$$

A somewhat coarser but related measure is the dimension of a pair $(F, G)$ :

Definition 10 For $F \subseteq G$, an element $\in G$ is enforced in $(F, G)$ if $F<\operatorname{opt}(G-\{e\})$. Otherwise it is free. The domain of $(F, G)$ is the set of free elements, i.e.

$$
\mathcal{D}(F, G):=\left\{e \in G \mid \exists F^{\prime} \subseteq G-\{e\}, F^{\prime} \leq F\right\} .
$$

Finally, the dimension of $(F, G)$ is the size of its domain,

$$
\operatorname{dim}(F, G):=|\mathcal{D}(F, G)|
$$

As an example consider Figure 3: subsets of $G$ are visualized by elements of $\{\boldsymbol{\square}, \square\}^{|G|} .(F, G)$ enforces the element 2 while $\left(F^{\prime}, G\right)$ enforces 2 and 5 . Consequently, $\mathcal{D}(F, G)=\{1,3,4,5\}$, $\mathcal{D}\left(F^{\prime}, G\right)=\{1,3,4\}$ and $\operatorname{dim}(F, G)=4, \operatorname{dim}\left(F^{\prime}, G\right)=3$. Note that $\operatorname{rank}(F, G) \leq 2^{\operatorname{dim}(F, G)}-1$.

The following monotonicity Lemma is an immediate consequence of the definitions and (although quite obvious) forms the background of the analysis in the next stage.

Lemma 11

(i) If $F^{\prime} \leq F$ then $\mathcal{D}\left(F^{\prime}, G\right) \subseteq \mathcal{D}(F, G)$.

(ii) If $F \subseteq G \subseteq G^{\prime}$ then $\mathcal{D}(F, G) \subseteq \mathcal{D}\left(F, G^{\prime}\right)$. 


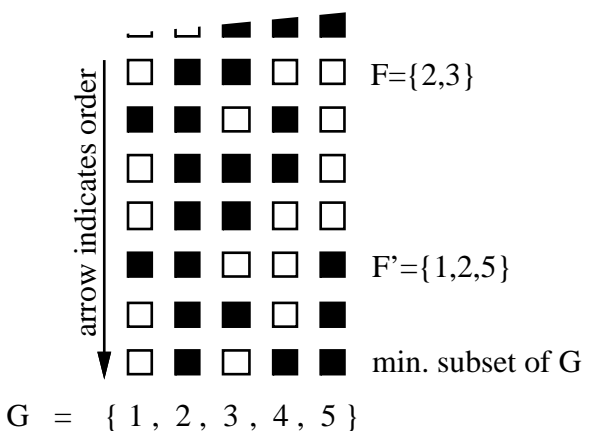

Figure 3: Part of an AOP on 5 elements

The basic algorithm. Now we are able to describe a first basic version of our algorithm. We feel that the main idea behind the subexponential analysis can be explained most clearly by assuming that the following subroutine is available:

\begin{tabular}{|c|c|}
\hline \multicolumn{2}{|c|}{ SAMPLE_DOMAIN $(F, G)$} \\
\hline 1 & if $\mathcal{D}(F, G)=\emptyset$ \\
\hline 2 & then error "empty" \\
\hline 3 & else choose a random $e$ from $\mathcal{D}(F, G)$ \\
\hline 4 & choose $F_{e} \leq F$ with $F_{e} \subseteq G-\{e\}$ \\
\hline & $\operatorname{return}\left(e, F_{e}\right)$ \\
\hline
\end{tabular}

So Sample_Domain chooses an element which is free in $(F, G)$ at random and computes a corresponding 'witness' $F_{e}$. Note that the elements in $G-F$ are free with witness $F$; however, there may be other free elements which are not immediately accessible since they are hidden in $F$, and it is not clear whether one can find them efficiently. Nevertheless, let us assume for this stage that SAMPLE_Domain comes for free.

Using this subroutine, we can formulate a procedure AoP_SD $(F, G)$ to find opt $(G)$ in presence of estimate $F$ :

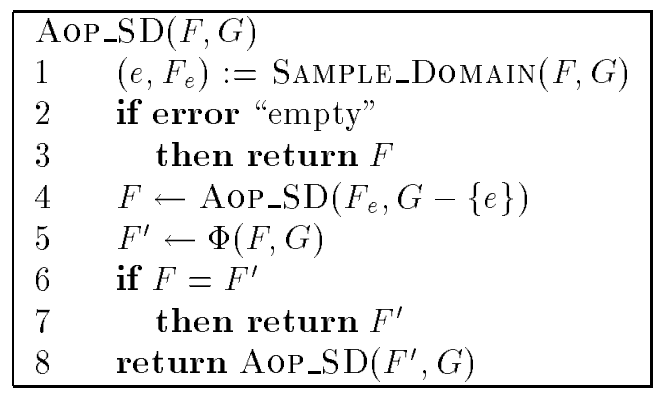

The analysis. Termination and Correctness easily follow by observing that the first recursive call solves a subproblem on a smaller set and in the second one we have $\operatorname{rank}\left(F^{\prime}, G\right)<\operatorname{rank}(F, G)$. For fixed $H$ let $T(k)$ be the worst case expected number of oracle queries performed in a call to Aop_SD $(F, G)$ with $\operatorname{dim}(F, G)=k$ and $G \subseteq H$. We get $T(0)=0$ and for $k>0$

Theorem 12

$$
T(k) \leq T(k-1)+1+\frac{T(k-1)+\ldots+T(0)}{k} .
$$


Proof: First of all, it is not hard to see that $T$ is monotone in $k$. Now we can argue as follows: if $(F, G)$ has dimension $k$ then $\operatorname{dim}\left(F_{e}, G-\{e\}\right) \leq k-1$, since

$$
\mathcal{D}\left(F_{e}, G-\{e\}\right) \cup\{e\} \subseteq \mathcal{D}\left(F_{e}, G\right) \subseteq \mathcal{D}(F, G) .
$$

This implies that the expected number of oracle queries necessary in the first recursive call is bounded by $T(k-1)$. Another query is done in line 5 ; the last term finally gives a bound for the second recursive call. To see this, consider $\mathcal{D}(F, G)=\left\{e_{1}, \ldots, e_{k}\right\}$, ordered in such a way that

$$
\operatorname{opt}\left(G-\left\{e_{1}\right\}\right) \geq \operatorname{opt}\left(G-\left\{e_{2}\right\}\right) \geq \ldots \geq \operatorname{opt}\left(G-\left\{e_{k}\right\}\right) .
$$

If $e$ is chosen to be $e_{i}$ in line 3 , we get

$$
F^{\prime}<\operatorname{opt}\left(G-\left\{e_{j}\right\}\right), \text { for all } j \leq i,
$$

so by Definition $10, e_{1}, \ldots, e_{i}$ will be enforced in $\left(F^{\prime}, G\right)$. This means

$$
\mathcal{D}\left(F^{\prime}, G\right) \subseteq \mathcal{D}(F, G)-\left\{e_{1}, \ldots, e_{i}\right\},
$$

so $\operatorname{dim}\left(F^{\prime}, G\right) \leq k-i$ and an expected number of no more than $T(k-i)$ oracle queries is performed in AoP_SD $\left(F^{\prime}, G\right)$. Since $i$ is equally likely to be any number between 1 and $k$, we obtain the desired average.

The heart of the argument is the fact that on the average the dimension halves when going from pair $(F, G)$ to $\left(F^{\prime}, G\right)$, and this pursues a method first applied by Kalai and more generally by Matoušek, Sharir \& Welzl.

In order to find an explicit bound for $T(k)$, we majorize $T(k)$ by $t(k)-1$ where $t(k)$ satisfies

$$
t(k)=t(k-1)+\frac{1}{k} \sum_{i=0}^{k-1} t(i)
$$

with $t(0)=1$.

\section{Lemma 13}

$$
t(k)=\sum_{i=0}^{k}\left(\begin{array}{l}
k \\
i
\end{array}\right) \frac{1}{i !} .
$$

Proof: An easy way to see this goes via a nice combinatorial interpretation of $t(k)$ (suggested by P. Flajolet): Consider a permutation $\pi \in S_{k}$. A subset $R \subseteq\{1, \ldots, k\}$ is called an increasing chain in $\pi$ iff $\forall x, y \in R: x<y$ implies $\pi(x)<\pi(y)$. Denote by $s(\pi)$ the number of increasing chains in $\pi$. Then $t(k)=E[s]$, the expected number of increasing chains in a random permutation $\pi \in S_{k}$. The proof of this fact is by induction. For $k=0$ we have one increasing chain, namely the empty set. Assume $k>0$; by the inductive hypothesis the expected number of increasing chains not containing $k$ is $t(k-1)$. The ones containing $k$ are in one-to-one correspondence with the increasing chains in $\left\{1, \ldots, \pi^{-1}(k)-1\right\}$, whose expected number is $t\left(\pi^{-1}(k)-1\right)$. Since $\pi^{-1}(k)$ is equal to $i$ with probability $1 / k$, for any $i \in\{1, \ldots, k\}$, we recover the original recurrence for $t(k)$. On the other hand,

$$
\begin{aligned}
t(k)=E[s] & =\sum_{R} \operatorname{Prob}(R \text { is an increasing chain }) \\
& =\sum_{i=0}^{k} \sum_{|R|=i} \operatorname{Prob}(R \text { is an increasing chain })=\sum_{i=0}^{k}\left(\begin{array}{c}
k \\
i
\end{array}\right) \frac{1}{i !},
\end{aligned}
$$

so the lemma follows. 


\section{Corollary 14}

$$
t(k) \leq e^{2 \sqrt{k}}
$$

Proof:

$$
t(k)=\sum_{i=0}^{k}\left(\begin{array}{c}
k \\
i
\end{array}\right) \frac{1}{i !} \leq \sum_{i=0}^{k} \frac{k^{i}}{i !^{2}}=\sum_{i=0}^{k}\left(\frac{\sqrt{k}^{i}}{i !}\right)^{2} \leq\left(\sum_{i=0}^{k} \frac{\sqrt{k}^{i}}{i !}\right)^{2} \leq\left(\sum_{i=0}^{\infty} \frac{\sqrt{k}^{i}}{i !}\right)^{2}=e^{2 \sqrt{k}}
$$

The bound is almost tight; this will be discussed later. Concerning the performance of AoP_SD we get

Theorem 15 AoP_SD solves any $A O P(H,<, \Phi)$ with an expected number of at most $e^{2} \sqrt{|H|}-1$ oracle queries.

How to sample from the domain. In order to turn the procedure AoP_SD from the previous paragraph into a working algorithm, we have to do something about the subroutine SAMPLE_Domain. As we have already indicated, the way to deal with it will be to find a reasonably cheap way to 'approximate' it. The idea is simple: rather than sampling from the whole domain of a given pair $(F, G)$, we will identify a subset $D$ of the domain - along with the corresponding witnesses - and sample from $D$ only. After plugging in this version of SAMPLE_DomaIN, the expected performance of AoP_SD will drop off, depending on the size of $D$, which we assume to be a function $r=r(k)$ of $k=\operatorname{dim}(F, G)$; the recurrence of Theorem 12 then becomes

$$
T(k) \leq T(k-1)+\frac{T(k-1)+\ldots+T(k-r(k))}{r(k)} .
$$

This bound is exponential for $r(k)=O(1)$ but becomes better the closer $r(k)$ is to $k$. On the other hand, the larger $r(k)$ is, the harder is the task of finding the set $D$. A reasonable balancing is achieved by choosing $r(k)$ proportional to $k$, say $r(k)=\lceil c k\rceil$, for some fixed fixed $0<c<1$ (the exact value will be determined later). We will see that the additional effort to find $D \subseteq \mathcal{D}(F, G)$ of this size is small, and the algorithm basically preserves its expected performance as analyzed above.

To construct $D$ when called on a pair $(F, G)$, the algorithm proceeds incrementally; since we know that at least the elements in $G-F$ are free with witness $F$, we can start off by setting $D$ to $G-F$. In case $D$ is already large enough, we just sample from $D$ and proceed as before. Otherwise we will have to enlarge $D$ by at least one more free element hidden in $F$; to this end we will step through a sequence of improving oracle queries (in a way to be described in a minute), until a witness for a yet unknown free element is found (or we already end up in opt $(G)$ ).

Suppose that in the generic step certain elements in $G$ have already been identified as free in $(F, G)$, and we need to find another free one. Here is the way to do it: call the algorithm recursively with $\left(F^{\prime}, G\right)$, where $F^{\prime}$ is the current estimate, but supply an additional parameter $E$ that contains all the elements of $G$ whose status is yet unknown (note that $E \subseteq F^{\prime}$ ). This recursive call now has two ways to terminate: either it finds the desired solution opt $(G)$ or - while improving its estimate - discovers an $F^{\prime \prime} \subseteq G$ which fails to contain $E$. This, however, means that the elements in $E-F^{\prime \prime}$ have been uncovered as free elements with witness $F^{\prime \prime}$, so the call has accomplished its task. The key observation is that as long as $D$ is small, the set $E$ of elements with unknown status will be large, and since the recursive call with parameter $E$ terminates as soon as the first estimate $F^{\prime \prime}$ appears that is no longer wedged between $E$ and $G$, it actually operates only on $G-E$ instead of $G$, which makes it substantially cheaper (this method is a generalization of the idea behind the pivoting strategy Kalai uses in his LP algorithm). 
A working algorithm. The generic call will have three parameters $E \subseteq F \subseteq G$, where in the beginning $E=\emptyset$. Let us formulate the procedure $\operatorname{Aop}(E, F, G)$ that will either return opt $(G)$ or deliver an estimate $F^{\prime}<F$ with $E \nsubseteq F^{\prime}$ to a higher level in the recursion. The set $D$ is implicitly maintained, comments will refer to it.

\begin{tabular}{|c|c|c|}
\hline \multicolumn{2}{|c|}{$\operatorname{AOP}(E, F, G)$} & $\triangleright E \subseteq F \subseteq G ;$ returns opt $(G)$ or $F^{\prime}<F, E \nsubseteq F^{\prime}$ \\
\hline 1 & if $E=G$ & \\
\hline 2 & then return $\Phi(F, G)$ & $\triangleright$ either $\Phi(F, G)=E=F=G$ or $E \not \subset \Phi(F, G)$ \\
\hline 3 & $E^{\prime} \leftarrow F$ & $\triangleright E^{\prime}$ contains $E$ and elements of unknown status \\
\hline 4 & for all $e \in G-E^{\prime}$ & $\triangleright D:=G-E^{\prime}$, the initial free elements \\
\hline 5 & do $F_{e} \leftarrow F$ & $\triangleright$ set witness \\
\hline 6 & while $\left|G-E^{\prime}\right|<\lceil c|G-E|\rceil$ & $\triangleright D$ still to small, try to enlarge it \\
\hline 7 & do $F-\operatorname{Aop}\left(E^{\prime}, F, G\right)$ & \\
\hline 8 & if $E \not \subset F$ & $\triangleright$ return to higher level in recursion \\
\hline 9 & then return $F$ & \\
\hline 10 & if $E^{\prime} \not \subset F$ & $\triangleright$ new free element(s) found \\
\hline 11 & then for all $e \in E^{\prime}-F$ & \\
\hline 12 & do $F_{e} \leftarrow F$ & $\triangleright$ set witness \\
\hline 13 & $E^{\prime} \leftarrow E^{\prime} \cap F$ & $\triangleright$ update $E^{\prime}\left(D:=D \cup\left(E^{\prime}-F\right)\right)$ \\
\hline 14 & else return $F$ & $\triangleright$ line 7 has already computed opt $(G)$ \\
\hline 15 & choose a random $e \in G-E^{\prime}$ & $\triangleright$ sample from $D$ \\
\hline 16 & $F \leftarrow \operatorname{Aop}\left(E, F_{e}, G-\{e\}\right)$ & \\
\hline 17 & if $E \nsubseteq F$ & $\triangleright$ return to higher level in recursion \\
\hline 18 & then return $F$ & \\
\hline 19 & $F^{\prime} \leftarrow \Phi(F, G)$ & $\triangleright$ once we get here, $F=\operatorname{opt}(G-\{e\})$ \\
\hline 20 & if $E \not \subset F^{\prime}$ or $F=F^{\prime}$ & $\triangleright$ check both termination criteria \\
\hline 21 & then return $F^{\prime}$ & \\
\hline 22 & return $\operatorname{Aop}\left(E \cup\{e\}, F^{\prime}, G\right)$ & $\triangle$ repeat with better estimate \\
\hline
\end{tabular}

Termination of AoP follows by observing that the recursive calls solve smaller problems (measured in terms of $|G-E|)$. The correctness of the procedure is not obvious at first sight, but it suffices to inductively check the invariant that $E$ is contained in every estimate up to the terminating one. This may be doubtful in the recursive call of line 22, when $E$ is replaced with $E \cup\{e\}$; to see that this is justified, observe that if the procedure gets through to line 22 at all, the call in line 16 must have actually computed $F=\operatorname{opt}(G-\{e\})$; moreover, $F^{\prime}<F$. This, however, means that $e$ is enforced in $\left(F^{\prime}, G\right)$, so for every future estimate $F^{\prime \prime} \subseteq G$ with $F^{\prime \prime} \leq F^{\prime}$ we will have

$$
E \not F^{\prime \prime} \Leftrightarrow E \cup\{e\} \nsubseteq F^{\prime \prime},
$$

so the invariant is guaranteed.

It should be mentioned that the estimate $F_{e}$ plugged into the recursive call in line 16 may be worse than some estimates computed during the while-loop. The important property, however, is that $F_{e}$ is at least as good as the original $F$ we started with.

Towards the recurrence. The reader might wonder whether this algorithm really matches the rough idea described above. For example, we have promised to make $D$ as large as $\lceil c|\mathcal{D}(F, G)|\rceil$. This holds if $E=\emptyset$, because in this case after the while-loop

$$
|D| \geq\lceil c|G-E|\rceil=\lceil c|G|\rceil \geq\lceil c|\mathcal{D}(F, G)|\rceil,
$$

but if $|E|>0$, we might end up with a much smaller $D$. In this case, however, there are elements in $\mathcal{D}(F, G)$ (especially the ones in $E$ ) which are actually enforced in the sense that every estimate containing $E$ has to contain these elements as well, and we should no longer consider such elements as free in a recursive call with parameters $E, F$ and $G$. The following definition for triples takes care of that; it mimics Definition 10 for pairs. 
Definition 16 For $E \subseteq F \subseteq G, e \in G-E$ is enforced in $(E, F, G)$ if $F<\operatorname{opt}(E, G-\{e\}):=$ $\min _{<}\left\{F^{\prime} \mid E \subseteq F^{\prime} \subseteq \bar{G}-\{\bar{e}\}\right\}$, and $e$ is free otherwise. The free elements form the domain of $(E, F, G)$, i.e.

$$
\mathcal{D}(E, F, G):=\left\{e \in G-E \mid \exists F^{\prime} \leq F, E \subseteq F^{\prime} \subseteq G-\{e\}\right\} .
$$

The dimension of $(E, F, G)$ is the size of its domain, i.e.

$$
\operatorname{dim}(E, F, G):=|\mathcal{D}(E, F, G)| .
$$

This ensures that the domain contains exactly the elements which are candidates for being included in $D$ and guarantees that $|D| \geq\lceil c|\mathcal{D}(E, F, G)|\rceil$ after the while-loop.

The main recurrence. In order to bound the expected performance of AoP, we can set up a recurrence which will look similar to the one that holds for AoP_SD. However, it will include an additional term for the effort that is necessary to find the set $D$; even worse, it will depend on two parameters rather than one which makes it somewhat harder to solve. Nevertheless, it basically behaves like the one-parameter version and we will be able to establish a similar bound.

For fixed $H$ and $m \geq k$, let $T(m, k)$ be the worst-case expected number of oracle queries performed in a call to $\operatorname{Aop}(E, F, G)$ with size $|G-E|=m$ and $\operatorname{dimension} \operatorname{dim}(E, F, G)=k$, $G \subseteq H$. For a statement $A$ let $\chi_{A}$ be 1 if $A$ holds and 0 otherwise. We get $T(0,0)=1$ and for $m>0$ we have

\section{Theorem 17}

$$
T(m, k) \leq 2+\sum_{i=0}^{\lceil c m\rceil-1} T(i, \min (i, k))+\chi_{k \geq\lceil c m\rceil}\left(T(m-1, k-1)+\frac{1}{\lceil c m\rceil} \sum_{i=1}^{\lceil c m\rceil} T(m-1, k-i)\right) .
$$

Proof: The arguments are basically the ones of Theorem 12, so we omit the details. Again $T$ is monotone in $k$; there are at most 2 oracle queries at the top level. In the worst case, the while-loop may be executed once with every value of $i:=\left|G-E^{\prime}\right|$ between 0 and $\lceil\mathrm{cm}\rceil-1$, which amounts to the $\sum_{i=0}^{\lceil c m\rceil-1} T(i, \min (i, k))$ term (note that we implicitly use the obvious generalization of the monotonicity Lemma 11 to triples). If $k<\lceil\mathrm{cm}\rceil$, the algorithm will not be able to find the required number of free elements and thus cannot get beyond the while-loop. Otherwise, the triple processed in line 16 has size $m-1$ and dimension at most $k-1$ ( $e$ is no longer free) which gives the $T(m-1, k-1)$ term. Finally, the last term bounds the expected effort in line 22 , averaged over the random choice in line 15 .

Solving the recurrence. It turns out that the behavior of $T(m, k)$ is only quasi-polynomial in $m$, while the major contribution comes from $k$. Let us define two auxiliary functions:

$$
\begin{aligned}
f(k) & =f(k-1)+\frac{1}{\lceil c k\rceil} \sum_{i=1}^{\lceil c k\rceil} f(k-i), \\
g(m) & =g(m-1)+\sum_{i=0}^{\lceil c m\rceil-1} g(i) .
\end{aligned}
$$

with $f(0)=1$ and $g(0)=2$.

\section{Lemma 18}

$$
T(m, k) \leq f(k) g(m)-1
$$

Proof: we proceed by induction on $m$. For $m=0$ we have equality, so assume $m>0$. Then $T(m, k)$ is bounded by

$$
2+\sum_{i=0}^{\lceil c m\rceil-1} f(\min (i, k)) g(i)-\lceil c m\rceil+\chi_{k \geq\lceil c m\rceil}\left(f(k-1) g(m-1)-1+\frac{1}{\lceil c m\rceil} \sum_{i=1}^{\lceil c m\rceil} f(k-i) g(m-1)-1\right)
$$




$$
\begin{aligned}
& \leq f(k) \sum_{i=0}^{\lceil c m\rceil-1} g(i)+\chi_{k \geq\lceil c m\rceil} g(m-1)\left(f(k-1)+\frac{1}{\lceil c k\rceil} \sum_{i=1}^{\lceil c k\rceil} f(k-i)\right)+2-\lceil c m\rceil-2 \chi_{k \geq\lceil c m\rceil} \\
& =f(k)(g(m)-g(m-1))+\chi_{k \geq\lceil c m\rceil} g(m-1) f(k)+2 \chi_{k<\lceil c m\rceil}-\lceil c m\rceil \\
& \leq f(k) g(m)-g(m-1)\left(f(k)-\chi_{k \geq\lceil c m\rceil} f(k)\right)+2 \chi_{k<\lceil c m\rceil}-1 \\
& \leq f(k) g(m)-g(0)\left(f(0)-\chi_{k \geq\lceil c m\rceil} f(0)\right)+2 \chi_{k<\lceil c m\rceil}-1 \\
& =f(k) g(m)-1 .
\end{aligned}
$$

It remains to bound $f(k)$ and $g(m)$.

\section{Lemma 19}

$$
\begin{aligned}
g(m) & \leq 5 m^{\log _{1 / c} m+1}, \\
f(k) & \leq(k+1)^{\alpha} e^{2 \sqrt{k / c}}, \text { where } \alpha:=\frac{1-c}{2 c} .
\end{aligned}
$$

Proof: The function $g(m)$ is monotone, so we can argue that $g(m) \leq g(m-1)+\lceil\mathrm{cm}\rceil g(\lceil\mathrm{~cm}\rceil-1)$, which by expanding $g(m-1)$ yields

$$
g(m) \leq \sum_{i=1}^{m}\lceil c i\rceil g(\lceil c i\rceil-1)+g(0) \leq m\lceil c m\rceil g(\lceil c m\rceil-1)+2 \leq m^{2} g(\lceil c m\rceil-1)+2 .
$$

We have $g(m) \leq h(m)-1$ for

$$
h(m)=m^{2} h(\lceil\mathrm{~cm}\rceil-1)
$$

with $h(0)=5$. Now we see that for $m>0, h(m) \leq 5 m^{\log _{1 / c} m+1}$ which holds for $m=1$, and inductively we obtain

$$
h(m) \leq 5 m^{2}(\mathrm{~cm})^{\log _{1 / c} c m+1}=5 m^{2} m^{\log _{1 / c} m-1}=5 m^{\log _{1 / c} m+1} .
$$

In order to bound $f(k)$ we proceed by induction, noting that for $k=0$ the bound holds. Now assume $k>0$ and recall that $\alpha=(1-c) / 2 c$. From the inductive hypothesis we get

$$
\begin{aligned}
f(k) & \leq k^{\alpha} e^{2 \sqrt{(k-1) / c}}+\frac{1}{\lceil c k\rceil} \sum_{i=1}^{\lceil c k\rceil}(k-i+1)^{\alpha} e^{2 \sqrt{(k-i) / c}} \\
& \leq k^{\alpha}\left(e^{2 \sqrt{(k-1) / c}}+\frac{1}{c k} \sum_{i=0}^{k-1} e^{2 \sqrt{i / c}}\right) .
\end{aligned}
$$

The sum can be estimated via the corresponding integral; we can write

$$
\sum_{i=1}^{k-1} e^{2 \sqrt{i / c}} \leq \int_{1}^{k} e^{2 \sqrt{x / c}} d x=\left[\sqrt{c} e^{2 \sqrt{x / c}}\left(\sqrt{x}-\frac{\sqrt{c}}{2}\right)\right]_{1}^{k}=\sqrt{c} e^{2 \sqrt{k / c}}\left(\sqrt{k}-\frac{\sqrt{c}}{2}\right)-\left(\sqrt{c} e^{2 \sqrt{1 / c}}-\frac{c}{2}\right) .
$$

For $c \leq 1$, the last term is at least one so that we get

$$
\sum_{i=0}^{k-1} e^{2 \sqrt{i / c}} \leq \sqrt{c} e^{2 \sqrt{k / c}}\left(\sqrt{k}-\frac{\sqrt{c}}{2}\right)
$$

This gives

$$
f(k) \leq k^{\alpha} e^{2 \sqrt{k / c}}\left(e^{2(\sqrt{(k-1) / c}-\sqrt{k / c})}+\frac{1}{\sqrt{c k}}-\frac{1}{2 k}\right) .
$$


Using $\sqrt{k-1}-\sqrt{k} \leq-\frac{1}{2 \sqrt{k}}$ we conclude that

$$
f(k) \leq k^{\alpha} e^{2 \sqrt{k / c}}\left(e^{-1 / \sqrt{c k}}+\frac{1}{\sqrt{c k}}-\frac{1}{2 k}\right),
$$

and by observing that $e^{x}$ is majorized by $1+x+\frac{x^{2}}{2}$ for negative $x$, we find that the term in brackets is upperbounded by

$$
1+\frac{1}{2 c k}-\frac{1}{2 k}=1+\frac{\alpha}{k}
$$

We finally obtain

$$
f(k) \leq k^{\alpha} e^{2 \sqrt{k / c}}\left(1+\frac{\alpha}{k}\right) \leq(k+1)^{\alpha} e^{2 \sqrt{k / c}} .
$$

It takes some more effort to show that $f(k)=\Theta\left(k^{-1 / 4} e^{2 \sqrt{k / c}}\right)$ if $c$ is a constant, so our simple estimate loses only slightly more than a $\sqrt[4]{k}$ factor if $c$ is close to 1 (if $c=1$, we recover the bound of Corollary 14).

We have proved that the expected number of oracle queries performed by algorithm AoP when called on a triple $(E, F, G)$ of size $m$ and dimension $k$, is bounded by

$$
T(m, k) \leq 5(k+1)^{(1-c) / 2 c} e^{2 \sqrt{k / c}} m^{\log _{1 / c} m+1}
$$

for any fixed $0<c<1$. Setting $z:=1 / c-1$ and rewriting the expression shows that

$$
T(n, n) \leq 5 n e^{2 \sqrt{n+z n}+\ln ^{2} n / \ln (1+z)+\ln (n+1) z / 2}
$$

queries are sufficient on the average to solve an Abstract Optimization Problem on a set $H$ with $n$ elements. By easy calculations, we see that the value $z_{0}$ minimizing this bound satisfies

$$
z_{0}=\frac{\ln n}{\sqrt[4]{n}}(1+o(1))
$$

so a reasonable choice for $z$ is $z=\ln n / \sqrt[4]{n}$. Exploiting that $\ln (1+z) \geq z-\frac{z^{2}}{2}$ for positive $z$ gives

$$
\frac{\ln ^{2} n}{\ln (1+z)} \leq \frac{\ln ^{2} n}{z-\frac{z^{2}}{2}}=\frac{\ln ^{2} n}{z}+\frac{\ln ^{2} n}{2-z} \leq \sqrt[4]{n} \ln n+\ln ^{2} n .
$$

This derivation holds for $z \leq 1$; in case $z>1$, the inequality follows directly. From the mean value theorem we get

$$
\sqrt{n+z n} \leq \sqrt{n}+\frac{z}{2} \sqrt{n} \leq \sqrt{n}+\frac{1}{2} \sqrt[4]{n} \ln n
$$

This leads to an overall upper bound of

$$
T(n, n) \leq 5 n e^{2 \sqrt{n}+2 \sqrt[4]{n} \ln n+\ln n\left(1+\frac{1}{2} \ln (n+1) / \sqrt[4]{n}\right)} .
$$

Theorem 20 An Abstract Optimization Problem $(H,<, \Phi)$ with $|H|=n$ can be solved with an expected number of no more than

$$
e^{2 \sqrt{n}+2 \sqrt[4]{n} \ln n+\ln n\left(2+\frac{1}{2} \ln (n+1) \sqrt[4]{n}\right)+\ln 5}=e^{2 \sqrt{n}+O(\sqrt[4]{n} \ln n)}
$$

oracle queries. 


\section{Discussion}

We have given an $e^{(2+o(1)) \sqrt{n}}$ expected time randomized algorithm for Abstract Optimization Problems on an $n$-element set $H$; the algorithm applies to the minimum spanning ball problem for $n$ points in $d$-space and to the problem of computing the minimum distance between two $d$-dimensional $n$-vertex (or $n$-facet) polyhedra, and for both problems we obtain the first subexponential bounds in $d$. As in the case of Linear Programming one can obtain a bound of $O\left(d^{2} n+e^{O(\sqrt{d \log d})}\right)$ for both problems by combining our result with the ones of [MSW] and [Cla2].

Recently, Ludwig [Lud] has shown that the problem of finding optimal strategies for simple stochastic games [Con] allows a subexponential solution (where he implicitly proved that the problem can be formulated as an AOP which allows direct application of the procedure AOP_SD from Subsection 4.2); unlike the AOPs we have discussed here, the simple stochastic game problem is of a more combinatorial rather than geometric flavor. In general, however, it seems that local optimization occurs most naturally in geometric settings.

To determine the randomized complexity of Abstract Optimization Problems itself is a challenging open problem. It seems that in order to substantially improve on the bound given here one would have to come up with a method that does not rely on the concept of the dimension of an estimate as a measure of progress during the algorithm. Any better upper bound would immediately imply better algorithms for the 'small' instances of the problems we have discussed and we believe that the ideas behind such progress would carry over to the 'large' instances. On the other hand, it is quite possible that there is a lower bound for AOPs that is in the range of the upper bound given here; however, so far we have not been able to establish any nontrivial lower

bound. A natural time class to consider when thinking about upper or lower bounds seems to be $2^{\log ^{r} n}$ for constant $r$.

\section{References}

[Cla1] K.L.Clarkson, Linear programming in $O\left(n 3^{d^{2}}\right)$, Inform. Process. Lett. 22(1986), 21-24.

[Cla2] K.L.Clarkson, Las Vegas algorithms for linear programming when the dimension is small, manuscript, 1989.

[Cla3] K.L.Clarkson, New applications of random sampling in computational geometry, Discrete and Computational Geometry 2 (1987), 195-222.

[CM] B.Chazelle, J.Matoušek, On linear-time deterministic algorithms for optimization in fixed dimension, manuscript, 1992.

[Con] A.Condon, The complexity of stochastic games, Information \& Computation 96(2) (1992), 203-224

[Dan] G.B.Dantzig, Maximization of a linear function of variables subject to linear inequalities, Activity Analysis of Production and Allocation, T.C.Koopman (Ed.) Cowles Commission Monograph 13, Wiley, New York, 1951, 339-347

[Dye1] M.E.Dyer, On a multidimensional search technique and its application to the euclidean one-center problem, SIAM J. Comput. 15(1986), 725-738

[Dye2] M.E.Dyer, A class of convex programs with applications to computational geometry, Proc. 8th annual Symp. on Comp. Geom., 1992

[Kal] G.Kalai, A subexponential randomized simplex algorithm, Proc. 24th ACM Symposium on Theory of Computing, 1992

[Kar] N.Karmarkar, A new polynomial-time algorithm for linear programming, Combinatorica 4 (1984), 373-395. 
[Kha] L.G.Khachiyan, Polynomial algorithm in linear programming, U.S.S.R. Comput. Math. and Math. Phys. 20 (1980), 53-72.

[KM] V.Klee and G.J.Minty, How good is the simplex algorithm, Inequalities III, O. Shisha (ed.) Academic Press, New York (1972), 159-175,

[Lud] W.Ludwig, A subexponential randomized algorithm for the simple stochastic game problem, Manuscript, 1992

[Meg] N.Megiddo, Linear programming in linear time when the dimension is fixed, J. Assoc. Comput. Mach. 31 (1984), 114-127.

[MSW] J.Matoušek, M.Sharir and E.Welzl, A subexponential bound for linear programming, Proc. 8th annual Symp. on Comp. Geom. (1992), 1-8

[Raj] V.T.Rajan, Optimality of the Delaunay triangulation in $\mathbb{R}^{d}$, Proc. 7th annual Symp. on Comp. Geom., 1991, 357-363

[Sch] A.Schrijver, Theory of linear and integer programming, Wiley, New York (1986)

[Sei] R.Seidel, Low-dimensional linear programming and convex hulls made easy, Discrete Comp. Geom. 6(1991), 423-434.

[SW] M.Sharir and E.Welzl, A combinatorial bound for linear programming and related problems, Proc. Symp. on Theoretical Aspects of Computer Science (1992)

[SY] K.Sekitani and Y.Yamamoto, A recursive algorithm for finding the minimum norm point in a polytope and a pair of closest points in two polytopes, Mathematical Programming, to appear

[Wel] E.Welzl, Smallest enclosing disks (balls and ellipsoids), New Results and New Trends in Computer Science, H.Maurer, Ed., 1991, Lecture Notes in Computer Science, to appear.

[Wol] P.Wolfe, The simplex method for quadratic programming, Econometrica 27, 382-398 